KIAS-P97007

JHU-TIPAC 97019

DFTT $71 / 97$

hep-ph/9711363

\title{
Coherence of neutrino oscillations in the wave packet approach
}

\author{
C. Giunti \\ INFN, Sezione di Torino, and Dipartimento di Fisica Teorica, Università di Torino, \\ Via P. Giuria 1, I-10125 Torino, Italy \\ C. W. Kim \\ Department of Physics \& Astronomy, The Johns Hopkins University, \\ Baltimore, MD 21218, USA, and \\ School of Physics, Korea Institute for Advanced Study, Seoul 130-012, Korea
}

\begin{abstract}
The temporal and spatial coherence widths of the microscopic process by which a neutrino is detected are incorporated in the quantum mechanical wave packet treatment of neutrino oscillations, confirming the observation of Kiers, Nussinov and Weiss that an accurate measurement of the energies of the particles participating in the detection process can increase the coherence length. However, the wave packet treatment presented here shows that the coherence length has an upper bound, determined by the neutrino energy and the mass-squared difference, beyond which the coherence of the oscillation process is lost.
\end{abstract}

PACS number: 14.60.Pq 
A complete understanding of neutrino oscillations must take into account the localization of microscopic processes by which a neutrino is produced and detected. This localization is appropriately described by a wave packet treatment of neutrino oscillations [1] 9] (different treatments are discussed in [10,11]). As the authors of [9] noticed, in the quantum mechanical wave packet approach presented in [⿴囗十 the dependence of the oscillation probability on the temporal and spatial coherence widths of the detection process was neglected. In this brief report, we wish to incorporate, in a simple and straightforward way, the temporal and spatial coherence widths of the detection process in the quantum mechanical wave packet description of neutrino oscillations and show that, as an immediate consequence of this, performing an accurate measurement of the energies of the particles involved in the detection process leads to an increase of the coherence length for neutrino oscillations, as was noticed for the first time in [6].

Let us consider a neutrino of flavor $\alpha$ produced by a weak interaction process at the origin of the space-time coordinates and detected at a distance $L$ after a time $T$ by a weak interaction process capable of detecting a neutrino of flavor $\beta$. As in [4], we describe the neutrino propagating between the production and detection processes with the timedependent state in the Schrödinger picture

$$
\left|\nu_{\alpha}(t)\right\rangle=\sum_{a} U_{\alpha a}^{*} \int \mathrm{d} p \psi_{a}\left(p ; p_{a}, \sigma_{p P}\right) e^{-i E_{a}(p) t}\left|\nu_{a}(p)\right\rangle,
$$

where $E_{a}(p)=\sqrt{p^{2}+m_{a}^{2}}$. This state is a superposition of mass eigenstate wave packet states (labeled by the index $a$ ) weighted by the complex-conjugated elements of the mixing matrix $U$ of the neutrino fields. For simplicity, we consider only one space dimension in the source-detector direction and we assume that the mass eigenstate wave functions in momentum space $\psi_{a}\left(p ; p_{a}, \sigma_{p P}\right)$ have the following gaussian form, which enables us to carry out an analytical calculation of the transition probability:

$$
\psi_{a}\left(p ; p_{a}, \sigma_{p P}\right)=\left(2 \pi \sigma_{p P}^{2}\right)^{-1 / 4} \exp \left[-\frac{\left(p-p_{a}\right)^{2}}{4 \sigma_{p P}^{2}}\right],
$$

where $p_{a}$ are the average momenta of the different mass eigenstates and $\sigma_{p P}$ is the momentum width of the wave packets. The average momenta $p_{a}$ are determined by the kinematics of the production process taking into account the masses $m_{a}$ of the mass eigenstates. We assume that all the mass eigenstates are extremely relativistic, i.e. $p_{a} \gg m_{a}$. In this case $\Delta E \simeq \Delta p$ and $\sigma_{p P}$ is determined by the minimum between the energy and momentum uncertainties of the production process (a possible dependence of the momentum widths from the index $a$ can be estimated to be very small and negligible for relativistic neutrinos). Hence, from the uncertainty principle it is clear that $\sigma_{p P}$ is determined by the maximum between the spatial and temporal coherence widths of the production process.

\footnotetext{
${ }^{1}$ Taking into account the spatial and temporal coherence widths of the production and detection processes, $x=0, t=0$ and $x=L, t=T$ are their average space-time coordinates.
} 
We assume that the gaussian wave functions (21) are sharply peaked around the corresponding average momentum, i.e. $\sigma_{p P} \ll E_{a}^{2} / m_{a}$, with $E_{a} \equiv E_{a}\left(p_{a}\right)$. Under this condition the energy $E_{a}(p)$ can be approximated by $E_{a}(p) \simeq E_{a}+v_{a}\left(p-p_{a}\right)$, where $v_{a}=p_{a} / E_{a}$ is the group velocity of each wave packet. In this case, the neutrino wave function in coordinate space $\left|\nu_{\alpha}(x, t)\right\rangle=\left\langle x \mid \nu_{\alpha}(t)\right\rangle$ is easily calculated after a gaussian integration to be given by

$$
\left|\nu_{\alpha}(x, t)\right\rangle=\left(2 \pi \sigma_{x P}^{2}\right)^{-1 / 4} \sum_{a} U_{\alpha a}^{*} \exp \left[-i E_{a} t+i p_{a} x-\frac{\left(x-v_{a} t\right)^{2}}{4 \sigma_{x P}^{2}}\right]\left|\nu_{a}\right\rangle,
$$

with the orthonormal states $\left|\nu_{a}\right\rangle$ belonging to the mass Fock space $\left(\left\langle\nu_{a} \mid \nu_{b}\right\rangle=\delta_{a b}\right)$. The width $\sigma_{x P}$ of the mass eigenstate wave packets in coordinate space is related to the momentum width $\sigma_{p P}$ by the uncertainty relation $\sigma_{x P} \sigma_{p P}=1 / 2$. From the considerations presented after Eq.(2) it is clear that the value of $\sigma_{x P}$ is given by the maximum between the spatial and temporal coherence widths of the production process.

Let us consider now the detection process which takes place at a distance $L$ and after a time $T$ from the origin of the space-time coordinates and is capable of detecting a neutrino with flavor $\beta$. In [4] the amplitude of the flavor changing process was obtained by projecting the state $\left|\nu_{\alpha}(L, T)\right\rangle$, which describes the propagating neutrino, on the flavor state $\left|\nu_{\beta}\right\rangle=\sum_{a} U_{\beta a}^{*}\left|\nu_{a}\right\rangle$. This procedure neglects the temporal and spatial coherence widths of the detection process. In order to take them into account, the detected neutrino must be described by a wave packet state analogous to that in Eq.(1):

$$
\left|\nu_{\beta}\right\rangle=\sum_{a} U_{\beta a}^{*} \int \mathrm{d} p \psi_{a}\left(p ; p_{a}, \sigma_{p D}\right)\left|\nu_{a}(p)\right\rangle .
$$

The value of the momentum width $\sigma_{p D}$ is given, for relativistic neutrinos, by the minimum between the energy and momentum uncertainties of the detection process. The state $\left|\nu_{\beta}\right\rangle$ does not evolve in time because it does not represent a propagating neutrino. The average momenta $p_{a}$ in the mass eigenstate gaussian wave functions describing the detected neutrino are the same as those of the corresponding mass eigenstate gaussian wave functions describing the neutrino propagating between production and detection. This property is a consequence of causality: after the average momenta $p_{a}$ have been determined by the kinematics of the production process, the mass eigenstates propagate between the two processes and determine the kinematics in the detection process. For example, the moduli of the average momenta $p_{a}$ of the mass eigenstate wave packets of a muon neutrino produced in pion decay at rest are fixed by the kinematics of the process. When this neutrino is detected, for example, by scattering with a nucleus at rest, each mass eigenstate determines with its average momentum $p_{a}$ and its mass $m_{a}$ a different value for the momenta of the recoil particles. Neutrino oscillations are observed if the different mass eigenstates are detected coherently, i.e. if the differences of the energies and momenta of the recoil particles corresponding to different mass eigenstates are smaller than the energy and momentum uncertainty of the detection process.

Taking into account that the detection process takes place at a distance $L$ from the origin of the coordinates, the wave function in coordinate space describing the detected neutrino is given by 


$$
\left|\nu_{\beta}(x-L)\right\rangle=\left(2 \pi \sigma_{x D}^{2}\right)^{-1 / 4} \sum_{a} U_{\beta a}^{*} \exp \left[i p_{a}(x-L)-\frac{(x-L)^{2}}{4 \sigma_{x D}^{2}}\right]\left|\nu_{a}\right\rangle,
$$

where $\sigma_{x D}$ is defined by the uncertainty relation $\sigma_{x D} \sigma_{p D}=1 / 2$. Hence, the value of $\sigma_{x D}$ is given by the maximum between the spatial and temporal coherence widths of the detection process.

The amplitude of the flavor changing process is given by the overlap

$$
A_{\alpha \beta}(L, T)=\int \mathrm{d} x\left\langle\nu_{\beta}(x-L) \mid \nu_{\alpha}(x, T)\right\rangle \text {. }
$$

The integral over $x$ is gaussian and leads to

$$
A_{\alpha \beta}(L, T)=\sqrt{\frac{2 \sigma_{x P} \sigma_{x D}}{\sigma_{x}^{2}}} \sum_{a} U_{\alpha a}^{*} U_{\beta a} \exp \left[-i E_{a} T+i p_{a} L-\frac{\left(L-v_{a} T\right)^{2}}{4 \sigma_{x}^{2}}\right],
$$

with

$$
\sigma_{x}^{2} \equiv \sigma_{x P}^{2}+\sigma_{x D}^{2}
$$

This relation is very important in that it clearly shows that the width $\sigma_{x}$ which determines the coherence of the flavor changing process depends on the spatial and temporal coherence widths of both the production and detection processes. The amplitude (0) has the same form as that in [4], with an important difference: the width $\sigma_{x}$ in [4] was determined only by the production process, whereas in Eq.(7) also the detection process is properly taken into account.

In order to calculate the oscillation probability, the mass eigenstate energies can be approximated by

$$
E_{a} \simeq E+\xi \frac{m_{a}^{2}}{2 E}
$$

where $E$ is the energy determined by the kinematics of the production process for a massless neutrino and $\xi$ is a dimensionless quantity that is determined by energy-momentum conservation in the production process to first order in $m_{a}^{2} / E^{2}$. The quantity $\xi$ is typically of order unity; for example, in neutrino production by pion decay at rest we have $\xi \simeq 0.2$. With this approximation we have $p_{a} \simeq E-(1-\xi) m_{a}^{2} / 2 E$ and $v_{a} \simeq 1-m_{a}^{2} / 2 E^{2}$. From Eqs.(可) and (9), the probability of $\nu_{\alpha} \rightarrow \nu_{\beta}$ transitions is given by

$$
\begin{aligned}
& P_{\alpha \beta}(L, T) \propto \sum_{a, b} U_{\alpha a}^{*} U_{\beta a} U_{\alpha b} U_{\beta b}^{*} \exp \left\{-i \frac{\Delta m_{a b}^{2}}{2 E}[\xi T+(1-\xi) L]\right\} \\
& \times \exp \left\{-\frac{\left(L-v_{a} T\right)^{2}+\left(L-v_{b} T\right)^{2}}{4 \sigma_{x}^{2}}\right\},
\end{aligned}
$$

with $\Delta m_{a b}^{2} \equiv m_{a}^{2}-m_{b}^{2}$.

In principle, $P_{\alpha \beta}(L, T)$ is a measurable quantity, but in all realistic experiments the distance $L$ is a fixed and known quantity, whereas the time $T$ is not measured and can have 
any value, because the source and the detector typically operate for times much longer than the oscillation times $4 \pi E / \Delta m_{a b}^{2}$. Therefore, the quantity that is measured in all experiments is the oscillation probability $P_{\alpha \beta}(L)$ at a fixed distance $L$, given by the time average of $P_{\alpha \beta}(L, T)$. After integrating over $T$ and imposing the normalization condition $\sum_{\beta} P_{\alpha \beta}(L)=$ 1 , we obtain

$$
P_{\alpha \beta}(L)=\sum_{a, b} U_{\alpha a}^{*} U_{\beta a} U_{\alpha b} U_{\beta b}^{*} \exp \left[-2 \pi i \frac{x}{L_{a b}^{\mathrm{osc}}}-\left(\frac{x}{L_{a b}^{\mathrm{coh}}}\right)^{2}\right] F_{a b},
$$

where

$$
L_{a b}^{\mathrm{osc}}=\frac{4 \pi E}{\Delta m_{a b}^{2}}, \quad L_{a b}^{\mathrm{coh}}=\frac{4 \sqrt{2} \sigma_{x} E^{2}}{\left|\Delta m_{a b}^{2}\right|}
$$

are the oscillation wavelengths and coherence lengths, respectively, and

$$
F_{a b}=\exp \left[-2 \pi^{2}(1-\xi)^{2}\left(\frac{\sigma_{x}}{L_{a b}^{\mathrm{osc}}}\right)^{2}\right] \text {. }
$$

Equation (11) contains, in addition to the usual expression for the neutrino oscillation probability (the first term in the exponential with $F_{a b}=1$ ), two additional factors, the first being the second term in the exponential that takes into account the coherence of the contributions of different mass eigenstate wave packets and the second being the additional factor $F_{a b}$. The factor $F_{a b}$ is equal to unity if $\sigma_{x} \ll\left|L_{a b}^{\mathrm{osc}}\right|$, which is a necessary condition for the observation of neutrino oscillations that must be satisfied by any realistic experiment. If this condition is not satisfied, the interference among different mass eigenstate wave packets is washed out and only a constant transition probability $P_{\alpha \beta}=\sum_{a}\left|U_{\alpha a}\right|^{2}\left|U_{\beta a}\right|^{2}$ can be observed.

It is important to notice that the integration over the oscillation time is a crucial step in the wave packet treatment of neutrino oscillations in space, because it allows us to eliminate the time degree of freedom, which is not measured in realistic experiments. This elimination is done at the classical level by integrating the probability, which is a classical quantity, and without any unphysical assumption on the energies and momenta of the mass eigenstates, which are determined by the production process [12]. An unphysical assumption is, for example, the imposition of equal energy to the different mass eigenstates, i.e. $\xi=0$, which would eliminate the time dependence of the oscillatory term in the probability (10).

From Eqs.(8) and (12) one can see that the coherence length $L_{a b}^{\mathrm{coh}}$ is proportional to $\sigma_{x} \equiv \sqrt{\sigma_{x P}^{2}+\sigma_{x D}^{2}}$. Hence, $L_{a b}^{\mathrm{coh}}$ is dominated by the largest among the temporal and spatial coherence widths of the production and detection process. In particular, a precise determination of the energies (or momenta) of the particles involved in the detection process implies a small $\sigma_{p D}$ and a large $\sigma_{x D}$, leading to a large coherence length $L_{a b}^{\text {coh }} \simeq 4 \sqrt{2} \sigma_{x D} E^{2} /\left|\Delta m_{a b}^{2}\right|$ (if $\sigma_{p D} \ll \sigma_{p P}$ ). Hence, as noted in [6], the coherence length can be increased by measuring accurately the energies of the particles participating in the detection process. However, even if, at least in principle, the coherence length can be increased without limit by an extremely precise measurement of the energies of the detection (or production) particles, the oscillations lose the coherence for $\sigma_{x} \gtrsim L_{a b}^{\text {osc }}$, because the factor $F_{a b}$ becomes important and has the effect of suppressing the interference of $\nu_{a}$ and $\nu_{b}$ for $\sigma_{x} \gg L_{a b}^{\text {osc }}$. Physically this is due to 
the fact that the spatial or temporal width of the detection (or production) process becomes larger than the oscillation length, leading to the washout of the oscillations]. In order to understand the reason of this washing-out let us consider, for example, the case in which the detection process has a small spatial coherence width and a large temporal coherence width that dominates the total coherence width $\sigma_{x}$. As shown by Eq.(10), in this case the interference between the mass eigenstates $a$ and $b$ is not suppressed if the average detection time $T$ does not differ from $L / v_{a}$ and $L / v_{b}$ by more than $\sim \sigma_{x}$. This is due to the fact that the modes of the detection process corresponding to the different mass eigenstates oscillate coherently during a time interval of width $\sigma_{x}$ around $T$, even when the mass eigenstate wave packets are far from $L$ [ ? Then it is clear that if $\sigma_{x}$ is larger that the oscillation length, the phase of the interference term depends crucially on the value of $T$. In this case, if $T$ is not measured the average of the transition probability over $T$ washes out the interference.

Therefore, using Eq.(12) one can see that the coherence length has the upper bound

$$
L_{a b}^{\mathrm{coh}} \lesssim E\left(L_{a b}^{\mathrm{osc}}\right)^{2}=\frac{16 \pi^{2} E^{3}}{\left(\Delta m_{a b}^{2}\right)^{2}}
$$

beyond which the coherence length loses its meaning, because the coherence of the process is lost for any value of the distances $L$.

In conclusion, we have shown that the temporal and spatial coherence widths of the detection process can easily be incorporated in the quantum mechanical wave packet treatment of neutrino oscillations. As a result, we confirm the observation presented in [6] that an accurate measurement of the energies (or momenta) of the particles participating in the detection process can increase the coherence length. However, the wave packet treatment presented here shows that the coherence length cannot be increased beyond the upper bound given by Eq.(14) without losing the coherence of the oscillation process.

\footnotetext{
${ }^{2}$ Notice that a similar effect is obtained if the distance $L$ from the source is not known and the time-dependent probability $P_{\alpha \beta}(T)$ is obtained by averaging $P_{\alpha \beta}(L, T)$ over $L$. Such a situation is realized, for example, in the calculation of the effects of neutrino oscillations in the early universe.

${ }^{3}$ Notice that if $\sigma_{x D}$ is dominated by the temporal coherence width of the detection process, the gaussian approximation in (5) for the wave function of the detected neutrino introduces an unphysical interaction for times smaller than the time of arrival of the propagating neutrino to the detector. In this case a more realistic approximation can be obtained by inserting in (5) and (6) a $\theta\left(x-L+\tilde{\sigma}_{x D}\right)$, where $\tilde{\sigma}_{x D}$ is the spatial coherence width of the detection process. This approximation requires a numerical solution of the integral in (6) and will be discussed in detail elsewhere [13].
} 


\section{REFERENCES}

[1] S. Nussinov, Phys. Lett. B 63, 201 (1976).

[2] S.M. Bilenky and B. Pontecorvo, Phys. Rep. 41, 225 (1978).

[3] B. Kayser, Phys. Rev. D 24, 110 (1981).

[4] C. Giunti, C.W. Kim and U.W. Lee, Phys. Rev. D 44, 3635 (1991).

[5] C. Giunti, C.W. Kim, J.A. Lee and U.W. Lee, Phys. Rev. D 48, 4310 (1993).

[6] K. Kiers, S. Nussinov and N. Weiss, Phys. Rev. D 53, 537 (1996).

[7] J.E. Campagne, Phys. Lett. B 400, 135 (1997).

[8] C. Giunti, C.W. Kim and U.W. Lee, hep-ph/9709494.

[9] K. Kiers and N. Weiss, hep-ph/9710289.

[10] J. Rich, Phys. Rev. D 48, 4318 (1993).

[11] W. Grimus and P. Stockinger, Phys. Rev. D 54, 3414 (1996).

[12] R.G. Winter, Lett. Nuovo Cimento 30, 101 (1981).

[13] C. Giunti, C.W. Kim and U.W. Lee, in preparation. 